\title{
Crioprotetores etileno glicol ou acetamida na viabilidade in vitro de espermatozoides congelados de ovinos
}

\author{
Ethylene glycol and acetamide cryoprotectants on in vitro viability of thawed ram spermatozoa
}

\author{
Ellen Cordeiro Bento da SilvaI Jobson Filipe de Paula Cajueiro ${ }^{\text {II }}$ Sildivane Valcácia Silva ${ }^{\text {III }}$ \\ Maria Madalena Pessoa Guerra ${ }^{I V}$
}

\section{RESUMO}

Objetivando-se avaliar o efeito de diferentes concentrações dos crioprotetores etileno glicol ou acetamida na viabilidade in vitro de espermatozoides congelados de ovinos, foram utilizados seis pools de sêmen de quatro reprodutores mestiços da raça Santa Inês. Após aprovação, o pool dos ejaculados foi diluído em Tris-gema, acrescido de glicerol ( $G 1=5 \%$; controle), etileno glicol (G2=3\%; G3=5\%) ou acetamida ( $G 4=2 \% ; G 5=7 \%)$, envasado em palhetas $\left(0,25 \mathrm{~mL} ; 100 \times 10^{6}\right.$ espermatozoides) e congelado. As amostras descongeladas $\left(37^{\circ} \mathrm{C} / 30\right.$ segundos) foram avaliadas quanto a motilidade progressiva (MP), vigor, integridade da membrana plasmática (iMP), potencial de membrana mitocondrial (PMM) e integridade do acrossoma (iAC). Constatou-se que a MP do G1 foi superior $(P<0,05)$ à dos $G 3, G 4$ e $G 5$, e que o vigor dos G1, G2 e G3 foi maior $(P<0,05)$ do que o dos G4 e G5. A iMP do $G 1$ foi superior $(P<0,05)$ à dos $G 2, G 3, G 4$ e $G 5$, assim como os $G 2$ e G3 foram superiores $(P<0,05)$ a $G 4$ e $G 5$. $O$ $P M M$ e a iAC não diferiram $(P>0,05)$ entre os grupos. Concluise que o etileno glicol (3 e 5\%) e a acetamida (2 e 7\%) não são eficazes para a proteção dos espermatozoides ovinos submetidos à congelação.

Palavras-chave: congelação, crioprotetores, ovino, sêmen.

\section{ABSTRACT}

Aiming to evaluate the effect of different concentrations of ethylene glycol or acetamide cryoprotectants on in vitro viability of ram frozen spermatozoa, were used six semen-pools from four Santa Inês crossbred males. After approval, the ejaculate-pool was diluted in Tris egg-yolk plus glycerol (G1=5\%; control), ethylene glycol ( $3 \%=G 2 ; G 3=5 \%)$ or acetamide (G4=2\%; G5=7\%), loaded in straws $(0.25 \mathrm{~mL}$; $100 \times 10^{6}$ sperm) and frozeng. Thawed samples $\left(37^{\circ} \mathrm{C} / 30\right.$ seconds) were evaluated for progressive motility (PM), vigor, plasma membrane integrity (PMi), mitochondrial membrane potential (MMP) and acrosome integrity (ACi). It was found that the PM of $G 1$ was higher $(P<0.05)$ than $G 3, G 4$ and $G 5$, and that the vigor of G1, G2 and G3 was higher $(P<0.05)$ than $G 4$ and $G 5$. The PMi of $G 1$ was higher $(P<0.05)$ than $G 2, G 3, G 4$ and $G 5$, as well as $G 2$ and $G 3(P<0.05)$ where high than $G 4$ and $G 5$. MMP and ACi were not different $(P>0.05)$ among groups. It is concluded that ethylene glycol (3 and 5\%) and acetamide (2 and $7 \%$ ) don't are effectives in protecting ram spermatozoa subjected to freezing.

Key words: freezing, cryoprotectants, ram, semen.

\section{INTRODUÇÃO}

A criopreservação do sêmen prolonga a longevidade dos espermatozoides pela redução de sua atividade metabólica, maximizando o uso dos animais de alto potencial genético e possibilitando o transporte do sêmen (SALAMON \& MAXWELL, 2000). Entretanto, as temperaturas usadas na criopreservação ocasionam danos ultra-estruturais, bioquímicos e funcionais às células (SALAMON \& MAXWELL, 2000), determinando baixos índices de fertilização após inseminação artificial (IA) (BRISOLA et al., 1999) e

\footnotetext{
IPrograma de Pós-graduação em Biotecnologia, Rede Nordeste de Biotecnologia, Universidade Federal Rural de Pernambuco (UFRPE), Recife, PE, Brasil.

"IClínica de Bovinos de Garanhuns, UFRPE, Garanhuns, PE, Brasil.

IIIPrograma de Pós-Graduação em Ciência Veterinária, UFRPE, Recife, PE, Brasil.

${ }^{\text {IV }}$ Departamento de Medicina Veterinária, UFRPE, 52171-900, Recife, PE, Brasil. E-mail: mpguerra@dmv.ufrpe.br. Autor para correspondência.
} 
tornando indispensável o uso dos crioprotetores espermáticos (SALAMON \& MAXWELL, 2000).

Dentre os crioprotetores penetrantes, o glicerol (peso molecular de 92,10) é o mais utilizado para a congelação de espermatozoides ovinos (SALAMON \& MAXWELL, 2000). Entretanto, o glicerol ocasiona danos osmóticos aos gametas, os quais dependem de fatores relacionados à espécie e ao indivíduo (ALVARENGA et al., 2005). Em decorrência de sua ação deletéria, muitos crioprotetores e suas combinações têm sido testados (PURDY, 2006), a fim de encontrar uma alternativa que diminua a toxicidade do glicerol (OKUDA et al., 2007), melhore a qualidade do sêmen criopreservado e os índices de nascimentos após IA (MORAES et al., 1998).

O etileno glicol, assim como o glicerol, é um crioprotetor alcoolico e de baixo peso molecular $(62,07)$. Esta substância tem sido testada na criopreservação dos espermatozoides (ALVARENGA et al., 2005), em decorrência de sua menor toxicidade e maior capacidade de penetração na célula (SILVA, 2007), o que possibilita a redução da concentração de sais no interior dos gametas e a proteção destes durante a criopreservação (MORAES et al., 1998). Todavia, os resultados obtidos após a criopreservação de sêmen com etileno glicol têm se mostrado contraditórios (SILVA, 2007), sendo necessários mais estudos para avaliar a viabilidade espermática em ovinos, utilizando métodos mais precisos e atuais de análise (ARRUDA et al., 2007).

As amidas formam o segundo grupo de crioprotetores penetrantes e, em virtude de seu baixo peso molecular $(59,07)$, induzem menos danos osmóticos, embora não existam estudos relatando a sua permeabilidade através da membrana plasmática espermática (ALVARENGA et al., 2005). Dentre as amidas, a acetamida se destaca por ter sido utilizada com sucesso na criopreservação de espermatozoides de espécies de difícil congelabilidade, como os de coelho a 1M (KASHIWAZAKI et al., 2006) e a $2 \%$ (OKUDA et al., 2007). Em espermatozoides ovinos, o único relato encontrado sobre o uso da acetamida é o de VIDAL et al. (2009), que verificaram efeito deletério desta substância nas concentrações de 3 e $5 \%$.

Na espécie ovina, é crescente a necessidade de melhorar a qualidade do sêmen congelado e os índices de nascimento após IA, fato que desperta o interesse em identificar crioprotetores mais eficazes que o glicerol e as concentrações necessárias destas substâncias para a obtenção de resultados satisfatórios. Desse modo, o objetivo deste estudo foi avaliar o efeito de diferentes concentrações de etileno glicol ou acetamida na viabilidade in vitro de espermatozoides congelados de ovinos.

\section{MATERIAL E MÉTODOS}

Para realização do estudo, cujo protocolo experimental foi aprovado pelo Comitê de Ética para Experimentação e Uso de Animais da Universidade Federal Rural de Pernambuco (CEEUA/DMV 080/2008), foram utilizados quatro reprodutores com fertilidade comprovada (1 a 2 anos de idade), mestiços da raça Santa Inês. Os animais foram criados em sistema de confinamento, sob iluminação natural (Recife-PE, Brasil; 8.0314 SL, $34.5252 \mathrm{~W}$ ), dieta composta de feno de tifton, concentrado (400g animal ${ }^{-1} \mathrm{dia}^{-1}$ ), sal mineral e água ad libitum.

As colheitas de sêmen foram realizadas com vagina artificial, utilizando uma fêmea como manequim, a intervalos de 48 horas, totalizando seis colheitas por reprodutor e 24 amostras de sêmen. Imediatamente após a colheita e aprovação dos ejaculados, procedeu-se à formação do pool de sêmen, o qual foi mantido em temperatura ambiente (sala climatizada a $\pm 28^{\circ} \mathrm{C}$ ) durante a realização das análises macroscópicas e microscópicas, segundo o Manual para exame andrológico e avaliação de sêmen animal do Colégio Brasileiro de Reprodução Animal (CBRA, 1998).

Durante a avaliação macroscópica do sêmen, apenas amostras com padrões considerados normais de volume (mL), aspecto e cor foram aprovadas. A análise microscópica foi realizada em microscópio de Contraste de Fase (Olympus, Tóquio, Japão) com aprovação das amostras que apresentaram turbilhonamento, motilidade progressiva (MP) e vigor espermático mínimos de 3 (05), $70 \%(0-100 \%)$ e $3(0-5)$, respectivamente. A concentração espermática foi determinada em Câmara de Neubauer (diluição 1:400, em formol citrato) e a morfologia espermática foi realizada pelo método de câmara úmida (diluição 1:400, em formol citrato), aprovando-se, respectivamente, as amostras com valores mínimos de 2x109 espermatozoides por mL e máximo de $20 \%$ de defeitos totais.

A análise de integridade da membrana plasmática (iMP) foi realizada com diacetato de carboxifluoresceína (DCF) e iodeto de propídio (IP), segundo a metodologia descrita por COLETO et al. (2002) e modificada por BATISTA (2009). Um total de 200 células foi analisado em microscópio de epifluorescência (Carl Zeiss, Germany, 400x), usando filtro de emissão DBP 580-630nm e excitação DBP 485/ $20 \mathrm{~nm}$, sendo classificadas como portadoras de membrana intacta, quando coradas em verde, ou danificada, quando corada em vermelho.

O potencial de membrana mitocondrial (PMM) foi determinado pela metodologia descrita por GUTHRIE \& WELCH (2006), com utilização do 
fluorocromo catiônico lipofílico JC-1. Um total de 200 células foi analisado em microscópio de epifluorescência (Carl Zeiss, Germany, 400x), em filtro de emissão LP 515nm e excitação BP 450-490nm, e classificadas como alto potencial de membrana mitocondrial, quando a peça intermediária fluoresceu em laranja, e baixo potencial de membrana mitocondrial, quando fluoresceu em verde.

A integridade do acrossoma (iAC) foi avaliada pela técnica de coloração Fluorescein isothiocyanate-conjugated aglutin (FITC-PNA), descrita por ROTH et al. (1998). Um total de 200 espermatozoides foi avaliado em microscópio de epifluorescência (Carl Zeiss, Göttingen, Germany, 1000x), usando filtro de emissão LP 515nm e BP 450-490nm para excitação. Os gametas foram classificados como portadores de acrossomas intactos, quando apresentavam a região acrossomal corada com fluorescência verde brilhante, ou acrossoma reagido, quando apresentavam uma faixa verde fluorescente na região equatorial da cabeça espermática ou não apresentavam fluorescência verde em toda região da cabeça.

Após análise, o pool dos ejaculados de cada repetição $(n=6)$ foi subdividido e diluído em Tris-gema de ovo $(67,2 \mathrm{~mL}$ de solução Tris, $20,0 \mathrm{~mL}$ de gema de ovo e 12,8mL de água miliQ; solução Tris: 3,605g de Tris-hidroximetilaminometano, 2,024g de ácido cítrico, 1,488g de frutose e 100mL de água miliQ; $\mathrm{pH}$ 6,8; EMBRAPA CNPC), acrescido dos crioprotetores, de acordo com os grupos experimentais: $\mathrm{G} 1=5 \%$ glicerol (controle; ACIBRA ${ }^{\circledR}$, São Paulo, Brasil); G2=3\% etileno glicol (VETEC, Rio de Janeiro, Brasil); G3=5\% etileno glicol; G4=2\% acetamida (Fluka, Chemical Co. Inc., Tóquio, Japão); G5=7\% acetamida.

Em seguida, as amostras de sêmen foram envasadas em palhetas $(0,25 \mathrm{~mL})$, em número de dez para cada grupo experimental, contendo $100 \times 10^{6}$ espermatozoides cada, e processadas em sistema de criopreservação convencional, constituído por geladeira e vapor de nitrogênio. Imediatamente após a refrigeração (redução da temperatura de $27^{\circ} \mathrm{C}$ a $5^{\circ} \mathrm{C}$, por 90 minutos), as palhetas foram mantidas a $5^{\circ} \mathrm{C}$ por 90 minutos, transferidas para a rampa de congelação $(3 \mathrm{~cm}$ de altura de nitrogênio líquido em caixa isotérmica, com distância de 4cm entre as amostras e o nível de nitrogênio; redução de $5^{\circ} \mathrm{C}$ a $-120^{\circ} \mathrm{C}$, por 15 minutos) e submersas e armazenadas em nitrogênio líquido $\left(-196^{\circ} \mathrm{C}\right)$.

No intervalo mínimo de 24 horas da congelação, amostras de sêmen (duas palhetas) foram descongeladas $\left(37^{\circ} \mathrm{C}\right.$ por 30 segundos) e avaliadas quanto à motilidade progressiva, vigor, integridade da membrana plasmática, potencial de membrana mitocondrial e integridade do acrossoma, conforme as técnicas descritas anteriormente.
Para a análise estatística, os valores das amostras de sêmen descongeladas foram submetidos à análise de variância (ANOVA) e as médias avaliadas pelo teste de comparação múltipla de Tukey, tendo sido os percentuais de motilidade progressiva, integridade da membrana plasmática, potencial de membrana mitocondrial e integridade do acrossoma transformados pelo arco seno (arco seno vP/100). Utilizou-se o programa INSTAT para Windows (versão 3.01), com delineamento inteiramente casualizado e significância de 5\%.

\section{RESULTADOS}

Após avaliação das amostras de sêmen congeladas em diluidor a base de Tris-gema, na presença de diferentes crioprotetores (Tabela 1), observou-se que o glicerol a 5\% (grupo controle) determinou maior motilidade progressiva $(\mathrm{P}<0,05)$ aos espermatozoides do que o etileno glicol a $5 \%$ e a acetamida a $2 \%$ e $7 \%$. Além disso, o vigor dos espermatozoides congelados com glicerol a 5\% e etileno glicol a $3 \%$ e $5 \%$ foi superior $(\mathrm{P}<0,05)$ ao da acetamida a $2 \%$ e $7 \%$.

O maior percentual de células com membrana plasmática íntegra $(\mathrm{P}<0,05)$ foi observado no grupo tratado com glicerol a 5\%, embora as amostras de sêmen criopreservadas com etileno glicol a 3\% e a 5\% também tenham determinado maior $(\mathrm{P}<0,05)$ manutenção deste parâmetro do que as de acetamida a $2 \%$ e a $7 \%$. Em contrapartida, não foram observadas diferenças significativas $(\mathrm{P}>0,05)$ para o potencial da membrana mitocondrial e integridade do acrossoma espermático.

\section{DISCUSSÃO}

Este estudo investigou o efeito de diferentes concentrações dos crioprotetores etileno glicol ou acetamida na viabilidade in vitro de espermatozoides congelados de ovinos. Para este fim, foi utilizada a concentração de $5 \%$ de glicerol, já consolidada na literatura para a congelação do sêmen ovino (SÁNCHEZ-PARTIDA et al., 1998; SALAMON \& MAXWELL, 2000), como grupo controle. O etileno glicol foi utilizado para confirmar se, em baixas concentrações (3\%), sua ação protetora é maior que a do glicerol e se, em concentrações elevadas (5\%), é tóxico aos espermatozoides. A acetamida foi testada a 2 e a $7 \%$ para identificar se nestas concentrações esta substância protege os espermatozoides ovinos melhor do que o glicerol.

A motilidade progressiva das amostras de sêmen congeladas com glicerol (5\%) e etileno glicol (3\% e 5\%) apresentaram valores compatíveis ao mínimo 
Tabela 1 - Parâmetros espermáticos de amostras de sêmen obtidas de reprodutores ovinos mestiços da raça Santa Inês e criopreservadas com glicerol (5\%), etileno glicerol (3 e 5\%) ou acetamida (2 e 7\%).

\begin{tabular}{|c|c|c|c|c|c|}
\hline \multirow{2}{*}{ Parâmetros espermáticos } & \multirow[b]{2}{*}{ G1 } & \multirow[b]{2}{*}{ G2 } & \multirow[b]{2}{*}{ G3 } & \multirow[b]{2}{*}{ G4 } & \multirow[b]{2}{*}{ G5 } \\
\hline & & & & & \\
\hline MP (\%) & $49,2 \pm 4,9^{\mathrm{a}}$ & $41,7 \pm 7,5^{\mathrm{ab}}$ & $33,3 \pm 10,3^{b}$ & $3,5 \pm 1,6^{\mathrm{c}}$ & $3,8 \pm 3,3^{c}$ \\
\hline Vigor (0-5) & $3,2 \pm 0,8^{\mathrm{a}}$ & $3,5 \pm 0,6^{\mathrm{a}}$ & $2,8 \pm 0,4^{\mathrm{a}}$ & $1,3 \pm 0,5^{\mathrm{b}}$ & $1,7 \pm 0,5^{\mathrm{b}}$ \\
\hline iMP (\%) & $38,2 \pm 16,4^{\mathrm{a}}$ & $17,5 \pm 7,2^{\mathrm{b}}$ & $15,3 \pm 6,2^{b}$ & $1,2 \pm 0,4^{\mathrm{c}}$ & $1,3 \pm 0,4^{\mathrm{c}}$ \\
\hline aPMM(\%) & $62,7 \pm 18,1$ & $69,1 \pm 22,5$ & $60,6 \pm 19,5$ & $75,9 \pm 24,9$ & $64,9 \pm 25,4$ \\
\hline iAC (\%) & $33,3 \pm 10,2$ & $39,8 \pm 13,3$ & $42,3 \pm 11,4$ & $34,2 \pm 10,6$ & $31,0 \pm 18,3$ \\
\hline
\end{tabular}

Letras diferentes na mesma linha indicam $\mathrm{P}<0,05$. MP=motilidade progressiva; iMP=integridade de membrana plasmática; aPMM=alto potencial de membrana mitocondrial; iAC=integridade de acrossoma; G1=glicerol 5\% (controle); G2=etileno glicol 3\%; G3=etileno glicol 5\%; G4=acetamida $2 \%$; G5=acetamida $7 \%$.

de $30 \%$, recomendados pelo Manual para exame andrológico e avaliação de sêmen animal do CBRA (1998), para sêmen descongelado. O mesmo foi observado para o vigor espermático dos grupos criopreservados com glicerol a $5 \%$ e etileno glicol a $3 \%$, cujos valores foram superiores a 3 .

Os resultados acima descritos podem ser justificados pelo fato da redução da motilidade espermática estar diretamente relacionada à perda de componentes intracelulares, lesões estruturais das membranas celulares (SILVA et al., 2006), alterações na energia disponível e danos nos elementos do axonema (WATSON, 1995). Corroborando à descrição anterior, verificou-se que o glicerol a $5 \%$ preservou melhor a integridade da membrana plasmática de espermatozoides ovinos do que o etileno glicol (3 e $5 \%$ ) e a acetamida (2 e 7\%), a qual foi deletéria aos gametas e determinou menor motilidade espermática.

A baixa capacidade crioprotetora da acetamida, possivelmente, resultou do estresse osmótico determinado pelo seu acúmulo no interior da célula, em virtude do baixo peso molecular. Tal suspeita é encorajada pelo fato de modificações nos meios diluidores poderem determinar distúrbios osmóticos (JULIANI \& HENRY, 2008), em que tanto osmolaridades altas quanto baixas são prejudiciais à sobrevivência dos espermatozoides, como consequência de alterações em suas estruturas e funções (LUZARDO et al., 2010).

Nesse contexto, é fortalecida a suspeita da existência de variações entre espécies e indivíduos à ação dos crioprotetores (SQUIRES et al., 2004). Isso porque, embora a acetamida tenha apresentado efeitos negativos sobre a congelação de espermatozoides ovinos neste estudo (2 e 7\%) e em estudo prévio (3 e $5 \%$ ) (VIDAL et al., 2009), assim como em espermatozoides equinos $(0,55 \mathrm{M})$ (SQUIRES et al., 2004), esta foi utilizada com sucesso no sêmen de coelho a $1 \mathrm{M}$ (KASHIWAZAKI et al., 2006) e a $2 \%$ (OKUDA et al., 2007).

Apesar do percentual de gametas com alto potencial de membrana mitocondrial ter se mantido semelhante entre os grupos experimentais, naqueles criopreservados com acetamida, os espermatozoides sofreram danos em suas demais estruturas, tornando inviável o uso na IA. Esse resultado pode ser explicado pelo fato da imobilidade das células, embora diretamente relacionada à redução da produção energética, pode estar associada à perda de componentes intracelulares, lesões estruturais das membranas celulares (SILVA et al., 2006) e danos nos elementos do axonema (WATSON, 1995).

Os percentuais de espermatozoides com acrossomas íntegros, em todos os grupos, foram considerados dentro do limite mínimo de 30\% para sêmen congelado de carneiro (EVANS \& MAXWELL, 1987). Tal achado corrobora BRIZOLA et al. (1999), ao demonstrarem não haver diferença significativa entre o glicerol $(0,72 \mathrm{M})$ e o etileno glicol $(0,5 \mathrm{M})$ para a proteção do acrossoma. Contudo, o etileno glicol (0,5M) é descrito como responsável por reduzir a concentração de sais no interior da célula e protegê-la dos efeitos nocivos da congelação (MORAES et al., 1998).

Em geral, foi observado que o etileno glicol (3 e 5\%) não preservou os parâmetros seminais de espermatozoides de ovinos, após a congelação, quando comparado ao glicerol (5\%), e que a acetamida (2 e 7\%) foi nociva às células espermáticas. A partir disso, é fortalecida a suspeita da existência de variações específicas e individuais à ação dos crioprotetores (SQUIRES et al., 2004), o que possivelmente está associada à variações na permeabilidade das membranas espermáticas, decorrentes das diferenças em sua composição (OKUDA et al., 2007).

A maior concentração de lipídeos na membrana, como colesterol, é responsável pelo 
aumento de sua rigidez e consequente redução da permeabilidade (LADHA, 1998), conforme pode ser observado em espermatozoides de coelhos (SALAMON \& MAXWELL, 2000). Em contrapartida, os espermatozoides de ovinos são ricos em ácidos graxos insaturados (SANOCKA \& KURPISZ, 2004), responsáveis pelo aumento de sua fluidez e permeabilidade (LADHA, 1998).

Em decorrência de sua composição, a membrana plasmática dos espermatozoides de carneiros possuem elevada permeabilidade ao glicerol (SALAMON \& MAXWELL, 2000), o que determina menor estresse osmótico e ação tóxica deste crioprotetor sobre as células (ALVARENGA et al., 2005). Desse modo, é provável que as menores barreiras existentes nas membranas dos espermatozoides ovinos, em associação aos baixos pesos moleculares do etileno glicol $(62,07)$ e da acetamida $(59,07)$, tenha favorecido a maior penetração destas substâncias no interior das células. Portanto, é possível que, neste estudo, a redução da motilidade progressiva, vigor e integridade da membrana plasmática ocasionadas especialmente pela acetamida, tenham sido determinadas pela ocorrência de distúrbios osmóticos, os quais comprometem a integridade dos espermatozoides (SNOECK et al., 2007).

\section{CONCLUSÃO}

O etileno glicol (3 e 5\%) e a acetamida (2 e $7 \%)$ não são eficazes para a proteção dos espermatozoides ovinos submetidos à congelação.

\section{AGRADECIMENTOS}

À Fundação de Amparo à Ciência e Tecnologia do Estado de Pernambuco (FACEPE), pela concessão de bolsa de mestrado e suporte financeiro. Ao Conselho Nacional de Desenvolvimento Científico e Tecnológico (CNPq), pelo apoio financeiro.

\section{REFERÊNCIAS}

ALVARENGA, M.A. et al. Amides as cryoprotectants for freezing stallion semen: a review. Animal Reproduction Science, Amsterdam, v.89, p.105-113, 2005. Disponível em: $<\mathrm{h} \mathrm{t} \mathrm{t} \mathrm{p} \mathrm{:} \mathrm{/} \mathrm{/} \mathrm{w} \mathrm{w} \mathrm{w.} \mathrm{s} \mathrm{c} \mathrm{i} \mathrm{e} \mathrm{n} \mathrm{c} \mathrm{e} \mathrm{d} \mathrm{i} \mathrm{re} \mathrm{c} \mathrm{t.} \mathrm{c} \mathrm{o} \mathrm{m} \mathrm{/}$ science?_ob=MImg\&_imagekei=B6T43-4GWBDX4-13\&_cdi=4963\&_user $=687346 \&$ \&ii $=$ S0378432005001971\& _orig=search\&_coverDate $=10 \% 2$ F31\%2F $2005 \&$ \& k = 999109998\&view=c\&wchhp=dGLzVzb-b\&ie=/sdarticle.pdf $>$. Acesso em: 10 abr. 2009. doi: 10.1016/ j.anireprosci.2005.07.001.

ARRUDA, R.P. et al. Biotécnicas aplicadas à avaliação do potencial de fertilidade do sêmen eqüino. Revista Brasileira de Reprodução Animal, Belo Horizonte, v.31, n.1, p.8-16,
2007. Disponível em: <http://www.cbra.org.br/pages/ publicacoes/rbra/download/RB108\%20Arruda\%20pag\%20816.pdf>. Acessado em: 04 maio, 2011.

BATISTA, A.M. Influência das técnicas de seleção Swimup e gradiente de densidade (Percoll ${ }^{\circledR}$ e Capripure) na viabilidade espermática de amostras criopreservadas de sêmen caprino. 2009. 53f. Dissertação (Mestrado em Ciência Veterinária) - Curso de Pós-graduação em Ciência Veterinária, Universidade Federal Rural de Pernambuco, PE.

BRISOLA, L.B.S. et al. Integridade das membranas plasmática, nuclear e mitocondrial de espermatozoides ovinos criopreservados com etileno glicol. Ciência Rural, Santa Maria, v.29, n.3, p.527-531, 1999. Disponível em: <http://www.scielo.br/pdf/cr/ v29n3/a25v29n3.pdf>. Acesso em: 03 jan. 2008.

COLETO, Z.F. et al. Avaliação do sêmen congelado de caprinos com drogas fluorescentes. Revista Brasileira de Medicina Veterinária, Rio de Janeiro, v.24, p.101-104, 2002.

GUTHRIE, H.D.; WELCH, G.R. Determination of intracellular reactive oxygen species and high mitochondrial membrane potential in Percoll-treated viable boar sperm using fluorescence-activated flow cytometry. Journal of Animal Science, Champaign, v.84, p.2089-2100, 2006. Disponível em: <http://jas.fass.org/cgi/reprint/84/8/2089>. Acesso em: 03 jan. 2008. doi: 10.2527/jas.2005-766.

JULIANI, G.C.; HENRY, M. Efeito do glicerol, etilenoglicol, acetamida e leite desnatado na criopreservação de espermatozóides eqüinos. Arquivo Brasileiro de Medicina Veterinária e Zootecnia, Belo Horizonte, v.60, n.5, p.11031109, 2008. Disponível em: <http://www.scielo.br/pdf/abmvz/ v60n5/10.pdf $>$. Acesso em: 22 fev. 2011.

KASHIWAZAKI, N. et al. Comparison of glycerol, lactamide, acetamide and dimethylsulfoxide as cryoprotectants of Japanese White Rabbit spermatozoa. Journal of Reproduction and Development, Tóquio, v.52, n.4, p.511-516, 2006. Disponível em: <http://www.jstage.jst.go.jp/article/jrd/52/4/511/_pdf>. Acesso em: 03 jan. 2008. doi: 10.1262/jrd.18008.

LADHA, S. Lipid heterogeneity and membrane fluidity in a highly polarized cell, the mammalian spermatozoon. Journal of Membrane Biology, Nova York, v.165, p.1-10, 1998. Disponível em: <http://www.springerlink.com/content/ kfbwtpwlqq834dyc/fulltext.pdf>. Acesso em: 29 dez. 2009. doi: $10.1007 / \mathrm{s} 002329900415$.

LUZARDO, B. et al. Osmolarity of coconut water (Cocos nucifera) based diluents and their effect over viability of frozen boar semen. American Journal of Animal and Veterinary Sciences, Nova York, v.5, n.3, p.187-191, 2010. Disponível em: <http://www.scipub.org/fulltext/AJAV/AJAV53187191.pdf $>$. Acesso em: 22 fev. 2011.

Manual para exame andrológico e avaliação de sêmen animal. 2.ed. Belo Horizonte: Colégio Brasileiro de Reprodução Animal (CBRA), 1998. 49p.

MORAES, C.N. et al. Criopreservação do sêmen ovino em pellets com etileno glicol. Ciência Rural, Santa Maria, v.28, n.2, p.287-292, 1998. Disponível em: <http://www.scielo.br/ pdf/cr/v28n2/a18v28n2.pdf $>$. Acesso em: 03 jan. 2008. doi: 10.1590/S0103-84781998000200018. 
OKUDA, Y. et al. Fertility of spermatozoa cryopreserved with $2 \%$ acetamide or glycerol through artificial insemination in the Japanese White Rabbits. Experimental Animals, Tóquio, v.56, n.1, p.29-34, 2007. Disponível em: <http:// www.jstage.jst.go.jp/article/expanim/56/1/29/_pdf $>$. Acesso em: 03 jan. 2008.

PURDY, P.H. A review on goat sperm cryopreservation. Small Ruminant Research, Amsterdam, v.63, p.215-225, 2006. Disponível em: <http://ddr.nal.usda.gov/bitstream/10113/33397/ 1/IND43848626.pdf $>$. Acesso em: 12 abr. 2009. doi: 10.1016/ j.smallrumres.2005.02.015.

ROTH, T.L. et al. Heterologous in vitro fertilization and sperm capacitation in an endangered African antelope, the ScimiltarHorned Oryx (Oryx dammah). Biology of Reproduction, Champaign, v.58, p.475-482, 1998. Disponível em: <http:// www.biolreprod.org/content/58/2/475.full.pdf+html>. Acesso em: 03 jan. 2008. doi: 10.1095/?biolreprod58.2.475.

SALAMON, S.; MAXWELL, W.M.C. Storage of ram semen. Animal Reproduction Science, Amsterdam, v.62, p.77-111, 2000. Disponível em: <http:// www.animalreproductionscience.com/article/S 0378 4320\%2800\%2900155-X/pdf>. Acesso em: 12 abr. 2009.

SÁNCHEZ-PARTIDA, L.G. et al. Effect of compatible solutes and diluent composition on the post-thaw motility of ram sperm. Reproduction, Fertility and Development, Melbourne, v.10, p.347-357, 1998. Disponível em: <http:// www.ncbi.nlm.nih.gov/pubmed/10355687>. Acesso em: $13 \mathrm{fev}$. 2012. doi: 10.1071/R98053.

SANOCKA, D.; KURPISZ, M. Reactive oxygen species and sperm cells. Reproductive Biology and Endocrinology, Londres, v.2, p.12-18, 2004. Disponível em: <http:// www.ncbi.nlm.nih.gov/pmc/articles/PMC400757/pdf/14777827-2-12.pdf $>$. Acesso em: 29 dez. 2009. doi: 10.1186/ 1477-7827-2-12.

SNOECK, P.P.N. et al. Efeito de diferentes diluidores sobre a viabilidade espermática pós-descongelação de sêmen eqüino.
Arquivo Brasileiro de Medicina Veterinária e Zootecnia, Belo Horizonte, v.59, n.1, p.56-64, 2007. Disponível em: <http://www.scielo.br/pdf/abmvz/v59n1/10.pdf>. Acesso em: 22 fev. 2011.

SILVA, A.R. Atualidades sobre a criopreservação do sêmen de cães. Revista Brasileira de Reprodução Animal, Belo Horizonte, v.31, n.1, p.119-127, 2007. Disponível em: <http://www.cbra.org.br/ pages/publicacoes/rbra/download/RB088\%20Silva\%20pag\%20119127.pdf>. Acesso em: 29 dez. 2009.

SILVA, A.F. et al. Uso de dimetil-formamida associada ou não ao glicerol na criopreservação de sêmen caprino. Revista Brasileira de Zootecnia, Viçosa, v.35, n.2, p.452-456, 2006. Disponível em: <http://www.scielo.br/pdf/rbz/v35n2/ a16v35n2.pdf>. Acesso em: 08 jun. 2009. doi: 10.1590/S151635982006000200016 .

SQUIRES, E.L. et al. Evaluation of alternative cryoprotectants for preserving stallion spermatozoa. Theriogenology, Stoneham, v.62, p.1056-1065, 2004. Disponível em: <http:// w w w. s c i e n c e d i r e c t . c o m / science?_ob=MImg\&_imagekey=B6TCM-4CB0R4X-2N\&_cdi $=5174 \&$ \&user $=687346 \&$ \&ii $=$ S0093691X04000809 \&_orig=search\&_coverDate $=09 \% 2$ F $15 \% 2$ F2004\&_sk $=$ 999379993 \& vi e w = c \& w c h p =d G L z V l b zSkzV\&md5=64d88160777ba6cad82606595229652a\&ie=/ sdarticle.pdf $>$. Acesso em: 03 jan. 2008. doi: 10.1016/ j.theriogenology.2003.12.024.

VIDAL, A.H. et al. Efeito da adição de acetamida na viabilidade in vitro de espermatozóides congelados ovinos. In: CONGRESSO BRASILEIRO DE REPRODUÇÃO ANIMAL, 18., 2009, Belo Horizonte. Anais... Belo Horizonte: CBRA, 2009. CD-ROOM.

WATSON, P.F. Recent developments and concepts in the cryopreservation of spermatozoa and the assessment of their post-thawing function. Reproduction, Fertility and Development, Melbourne, v.7, p.871-891, 1995. Disponível em: <http://www.ncbi.nlm.nih.gov/pubmed/8711221>. Acesso em: 13 de fev. 2012. doi: 10.1071/RD9950871. 